\title{
Publisher's Note: Unbiased charge oscillations in B-DNA: Monomer polymers and dimer polymers [Phys. Rev. E 92, 032725 (2015)]
}

K. Lambropoulos, M. Chatzieleftheriou, A. Morphis, K. Kaklamanis, M. Theodorakou, and C. Simserides

(Received 25 May 2016; published 6 June 2016)

DOI: 10.1103/PhysRevE.93.069902

This paper was published online on 24 September 2015 with errors in Fig. 5. Figure 5 has been replaced as of 24 May 2016. The figure is incorrect in the printed version of the journal; therefore for the benefit of the print readership, the figure and its caption are replicated below.
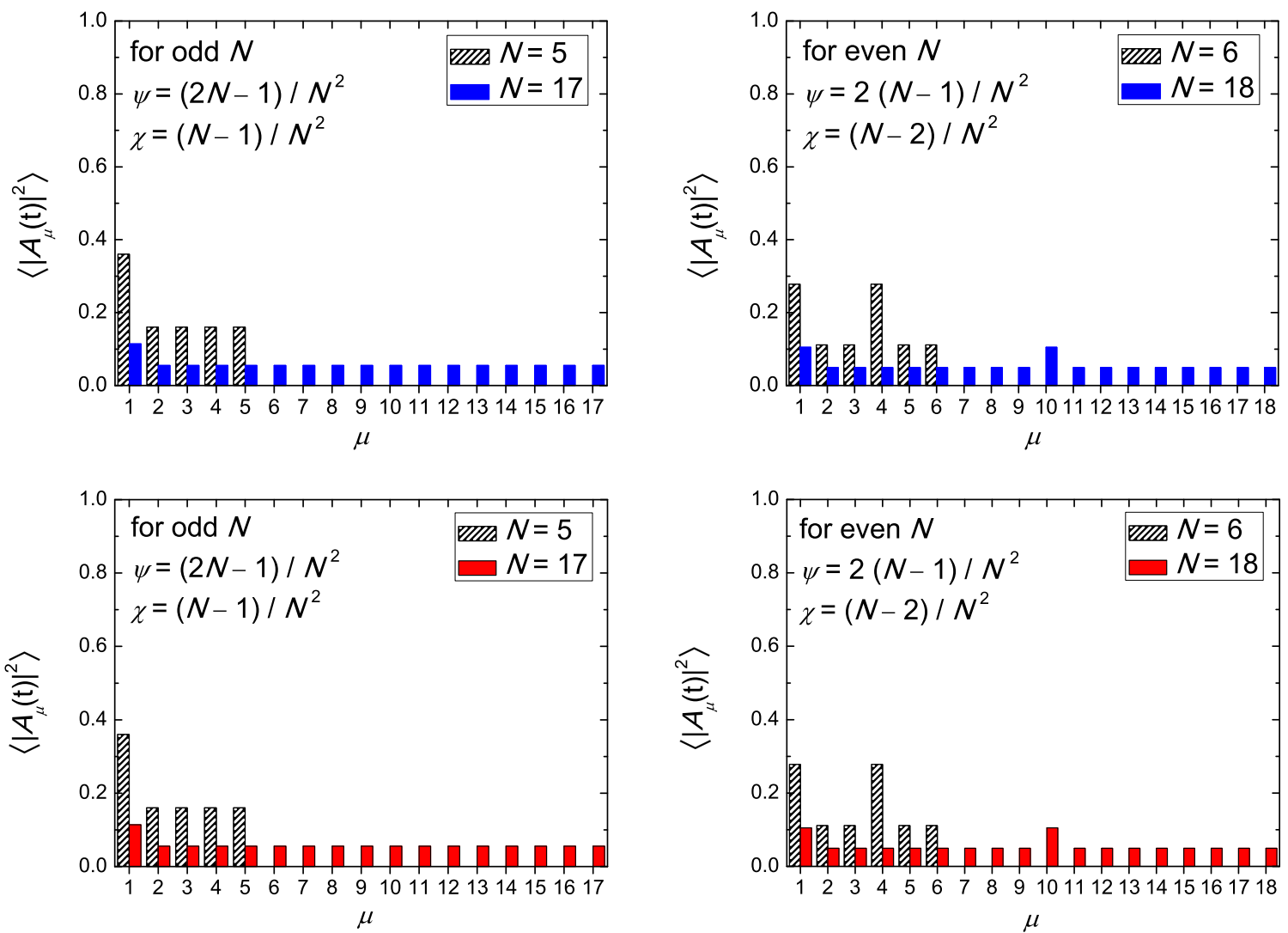

FIG. 5. Mean (over time) probabilities $\left\langle\left|A_{\mu}(t)\right|^{2}\right\rangle$ of type- $\alpha^{\prime}$ cyclic segments, if we initially place the carrier at the "first monomer." $\psi$ and $\chi$ denote $\left\langle\left|A_{\mu}(t)\right|^{2}\right\rangle$ at one of the favored monomers and at one of the rest monomers, respectively. We illustrate the situation for HOMO (first row) and LUMO (second row). The figures for cyclic poly(dG)-poly(dC) and cyclic poly(dA)-poly(dT) are identical. Left column: $N=5$ and $N=17$. Right column: $N=6$ and $N=18 .\left\langle\left|A_{\mu}(t)\right|^{2}\right\rangle$ follow Eqs. (40) and (41), i.e., depend only on $N$ and not on the on-site energies and the hopping integrals. If, on the contrary, we imagine to initially distribute the carrier probability equally among monomers, this initial equidistribution is conserved and no mean carrier movement occurs. 